\title{
Antibiotic-in-cyclodextrin-in-liposomes: formulation development and interactions with model bacterial membranes
}

Kalliopi-Kelli A. Vandera ${ }^{(\$)}$, Pietro Picconi ${ }^{(\$)}$, Margarita Valero ${ }^{(\&)}$, Gustavo González-Gaitano

${ }^{(\#)}$, Arcadia Woods ${ }^{(\$)}$, Nur Masirah M. Zain ${ }^{(\$)}$, Kenneth D. Bruce ${ }^{(\$)}$, Luke A. Clifton ${ }^{(+)}$, Maximilian W. A. Skoda ${ }^{(+)}$, Khondaker Miraz Rahman ${ }^{(\$)}$, Richard D. Harvey ${ }^{(*) *}, \underline{\text { Cécile A. Dreiss }}^{(\$) *}$

(\$) School of Cancer \& Pharmaceutical Science, Institute of Pharmaceutical Science, King's College London, Franklin-Wilkins Building, 150 Stamford Street, London, SE1 9NH, UK

(\&) Department of Physical Chemistry, University of Salamanca, ES E-37007, Salamanca, Spain

(\#) Department of Chemistry, University of Navarra, 31080, Pamplona, Spain

(+) Rutherford Appleton Laboratory, ISIS, 1-27, R3, Harwell Campus, Didcot, UK OX11 0QX

(¥) Department of Pharmaceutical Chemistry, University of Vienna, Althanstraße 14, Vienna, Austria 


\section{Supporting Information}

Table S 1. Physicochemical properties of PPA148 and rifampicin as estimated by ChemDraw software.

\begin{tabular}{|l|c|c|}
\hline \multicolumn{1}{|c|}{$\begin{array}{c}\text { Physicochemical } \\
\text { parameters }\end{array}$} & PPA148 & Rifampicin \\
\hline Molecular weight & 698.2 & 822.4 \\
$(\mathrm{~g} / \mathrm{mol})$ & 0.76 & 2.7 \\
\hline $\log$ & & \\
\hline $\operatorname{clogP}$ & 2.1 & - \\
\hline $\operatorname{logS}$ & -7.5 & 217.0 \\
\hline $\mathrm{S}(\mathrm{mg} / \mathrm{mL})$ & & \\
\hline
\end{tabular}


Aggregation and thermodynamic solubility in aqueous environment

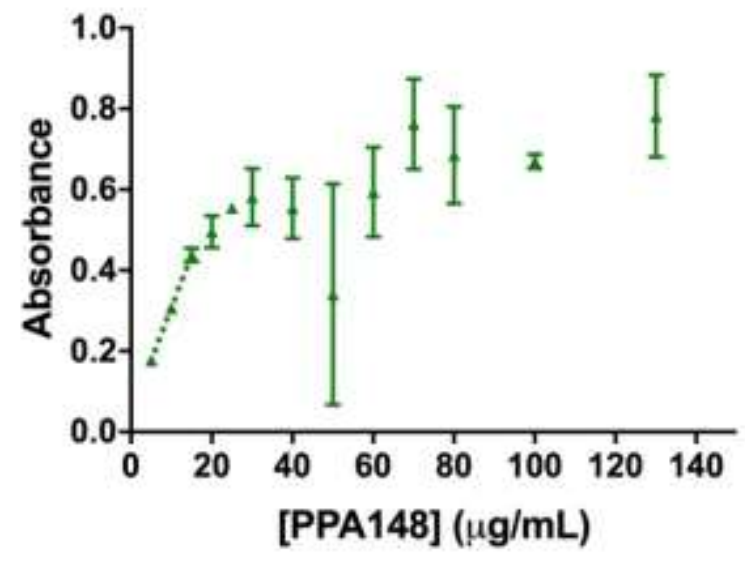

Figure S 1. Absorbance of increasing PPA148 concentration at a wavelength of $620 \mathrm{~nm}$ at $25{ }^{\circ} \mathrm{C}$. Linearity deviates for drug concentration above $15 \mu \mathrm{g} / \mathrm{mL}$.

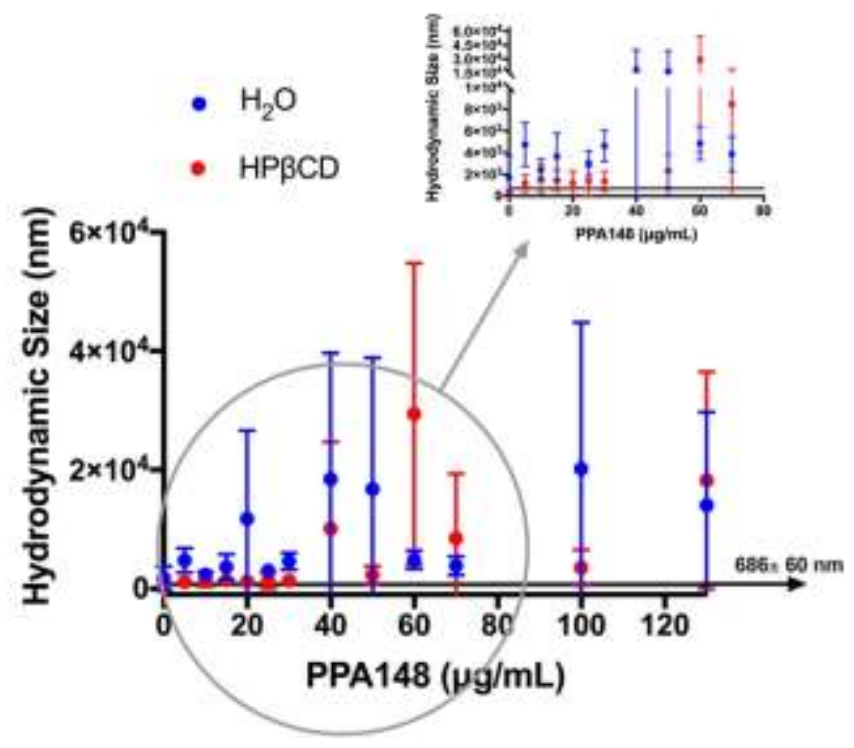

Figure S 2. Effect of increasing concentration of PPA148 on particle size when the drug is in $\mathrm{H}_{2} \mathrm{O}$ and $\mathrm{HP} \beta \mathrm{CD}$ at $25^{\circ} \mathrm{C}$. 
Stoichiometry of the drug/DIMEB complex by ${ }^{1} \mathrm{H}$ NMR: Job's Plot

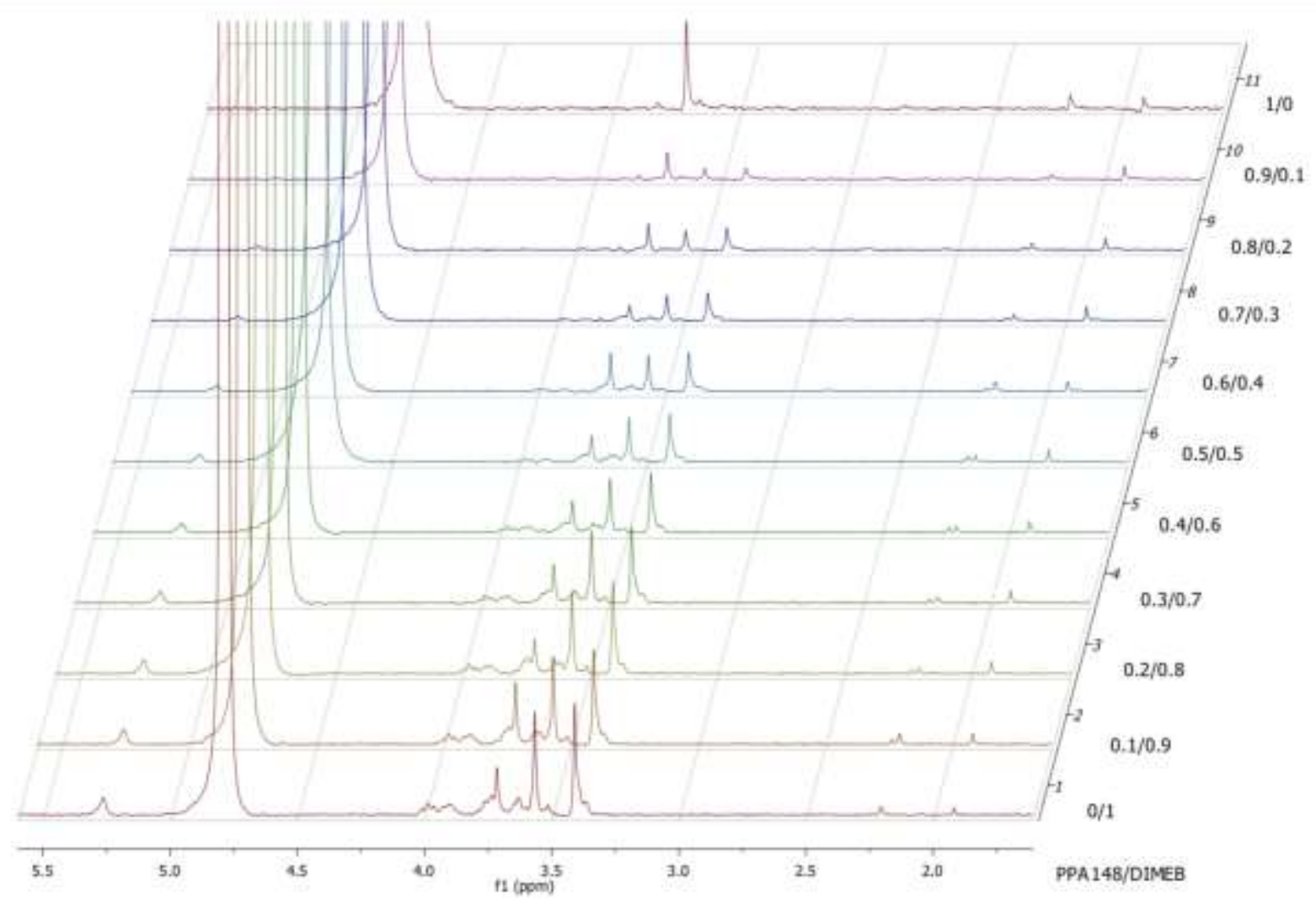

Figure S 3. ${ }^{1} \mathrm{H}$ NMR spectra used to plot Job's plot. 
PPA148/RAMEB complex encapsulated in fluidosomes

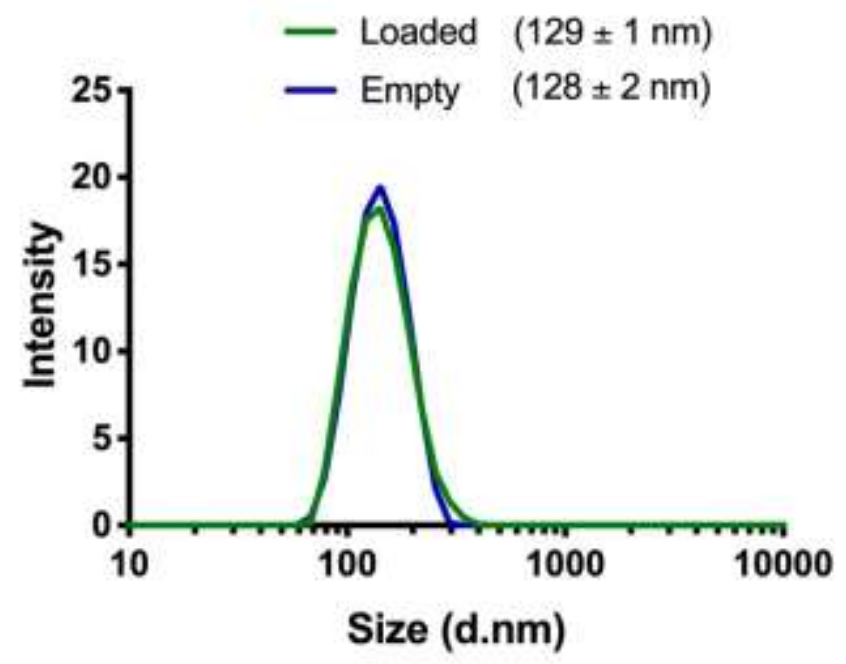

Figure S 4. Intensity distribution of empty DPPC/DMPG (18/1) liposomes in HEPES buffered saline (pH 7.2) using DLS.

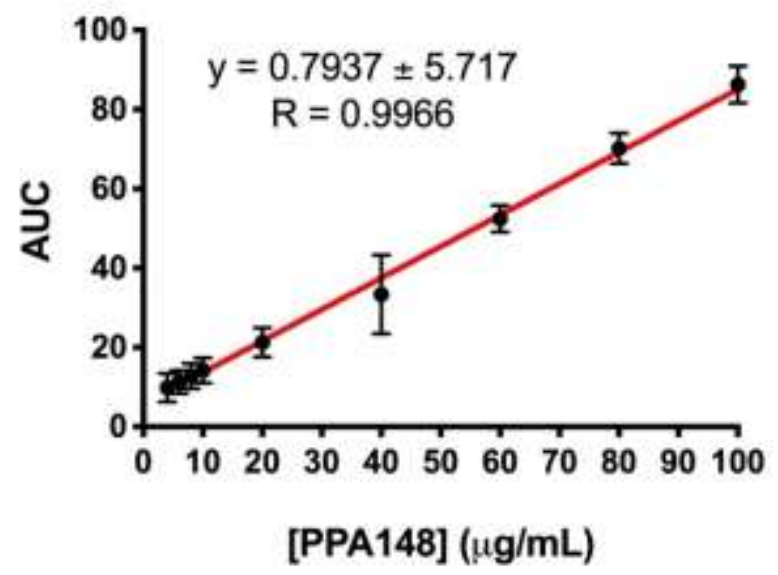

Figure S 5. Linear calibration curve of the area under the curve (AUC) of the UV spectrum of PPA148 for its quantification. The drug was extracted from the formulation and tested in 
ethanol/water mixture (80/20). The spectrum was measured in the range of 450-250 nm. Each standard sample was prepared and measured in triplicate.

\section{Stewart assay}

The determination of lipid concentration in the final formulation was achieved using the colorimetric Stewart assay. A calibration curve was established from standard DPPC solutions in chloroform $(1 \mathrm{mg} / \mathrm{mL})$. All standard samples were prepared by vigorously mixing the aqueous solution $(2 \mathrm{~mL})$ containing the reagent and the chloroform $(2 \mathrm{~mL})$ containing increasing concentrations of lipids $(0-0.05 \mathrm{mg} / \mathrm{mL})$. The mixtures were vigorously vortexed and then centrifuged (AllegraTM X-12 centrifuge, Beckman Coulter, UK) for 10 min at $1000 \mathrm{rpm}$ to separate the two phases. The organic phase was collected, and its absorbance measured at $467 \mathrm{~nm}$. A linear relationship was observed between the absorbance and the DPPC concentration in the complex and used as a calibration curve.

For the test samples, the suspension before separation and the 8 eluted aliquots from the Sephadex PD10 G25 size exclusion chromatography column were used to determine the lipid content in the starting and purified liposomes to detect any material loss during the process. The samples were lyophilized in an Alpha 1-2 LDplus freeze dryer (Martin Christ Gefriertrocknungsanlagen GmbH, Germany), connected with a Chemistry hybrid RC 6 pump (Vaccubrand GMBH, Germany) and then re-suspended in chloroform. A volume of $2 \mathrm{~mL}$ was transferred in chloroform $(2 \mathrm{~mL})$ which was added into the reagent solution to form the biphasic system. All tubes were vortexed as the standard samples and the DPPC concentration of the test tubes was interpolated from the calibration curve. All measurements were carried out in triplicate. The lipid content in each aliquot and the lipid loss in the third aliquot in the form of a percentage was calculated as follows: 


$$
\begin{aligned}
& \text { Lipid } \%=\frac{\text { Extrapolated lipids }\left(\frac{m g}{m L}\right)}{\text { Initial lipids }\left(\frac{m g}{m L}\right)} \times 100 \% \\
& \text { Lipid loss } \%=\frac{\text { Initial lipids }\left(\frac{m g}{m l}\right)-\text { Extrapolated lipids }\left(\frac{m g}{m l}\right)}{\text { Initial lipids }\left(\frac{m g}{m l}\right)} \times 100 \%
\end{aligned}
$$

\section{Fluidosomes stability}

The stability of fluidosomes was assessed by monitoring size over time. A low polydispersity index $(0.12 \pm 0.00$, compared to $0.22 \pm 0.02$ for the empty liposomes) was observed for liposomes. The effect of HEPES buffered saline ( $\mathrm{pH}$ 7.2) on the stability of fluidosomes was examined by diluting the initial lipid suspension up to 16 times from its original concentration $(4 \mathrm{mg} / \mathrm{mL})$. The size of all 4 diluted liposomal suspensions didn't change over dilution and time. 
(A)

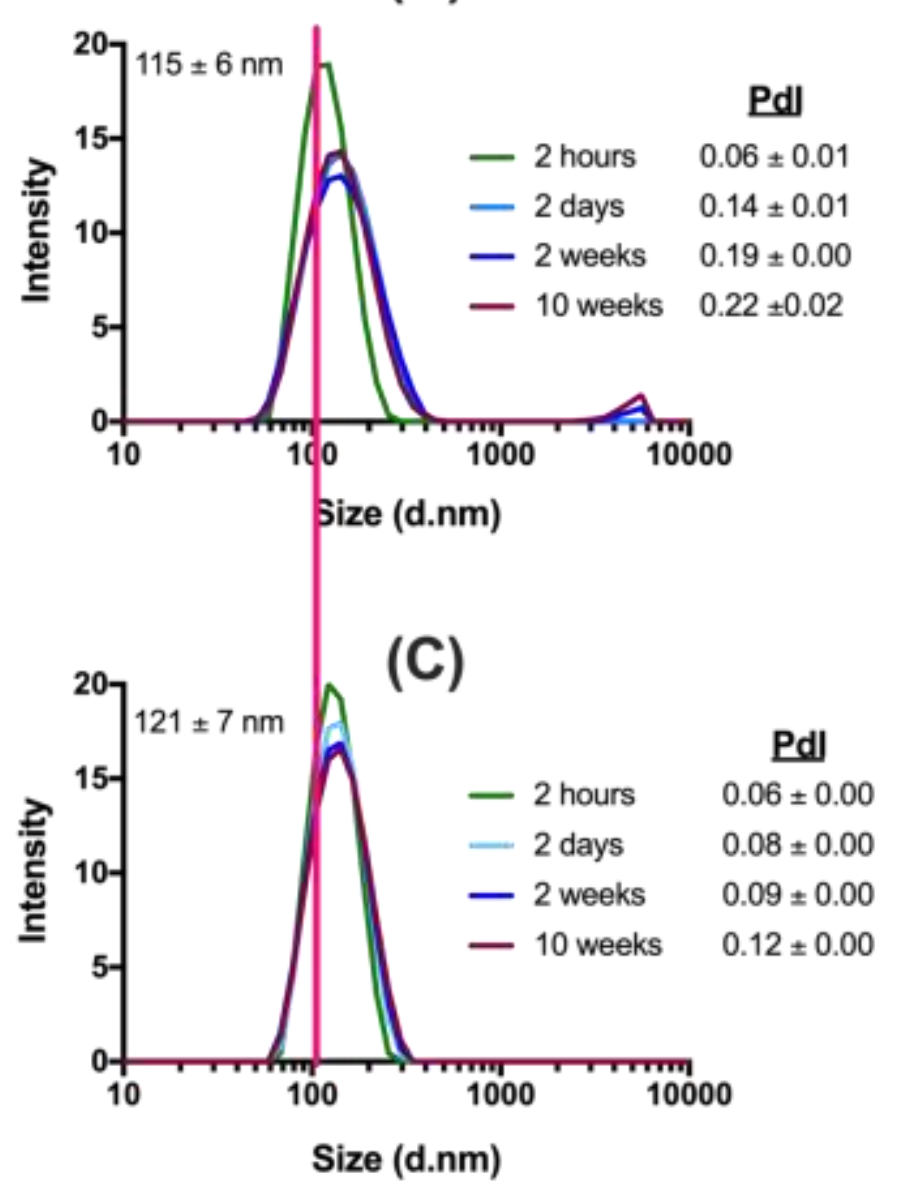

(B)
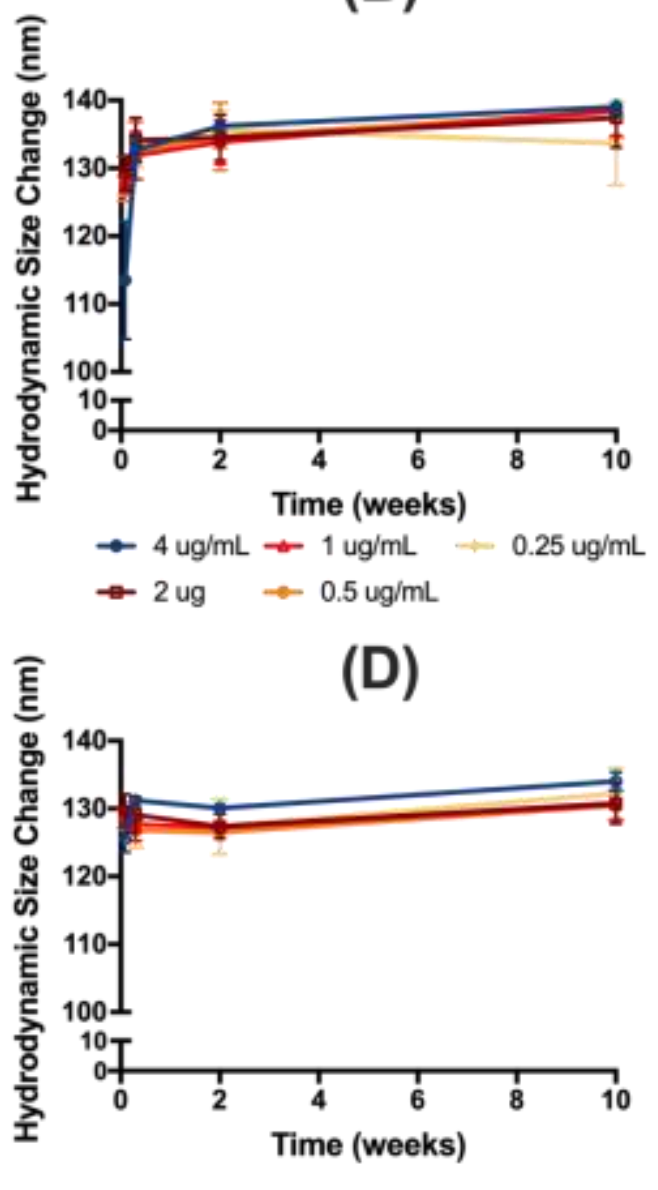

Figure S 6. Effect of DPPC/DMPG liposome integrity (A) without and (C) with 1\% RAMEB in terms of size and average intensity distribution profile of liposomes at a concentration of $4 \mathrm{mg} / \mathrm{mL}$ and temperature of $25^{\circ} \mathrm{C}$, measured by dynamic light scattering, over a period of 10 weeks. The samples were stored at $4{ }^{\circ} \mathrm{C}$ and tested between measurement intervals. The polydispersity index is presented at each time point. All sample were tested in triplicate. Effect of HEPES buffered saline $\mathrm{pH} 7.2$ on the size of liposomes $(1 \mathrm{mg} / \mathrm{ml})(\mathrm{B})$ in the absence and (D) presence of $1 \%$ RAMEB.

Adsorption isotherm of PPA148 
Affinity for the air/liquid interface, a measure of surface activity, was investigated at the molecular level using the adsorption isotherm technique (Figure S 7). PPA148 adsorbed at the air-water interface and reached an equilibrium maximum surface pressure of $13.3 \pm 1.3 \mathrm{mN} / \mathrm{m}$ within 2 hours. It was expected that the adsorption of PPA148 $(2 \mu \mathrm{g} / \mathrm{mL})$ at the air/liquid interface would be instantaneous because of the properties estimated by ChemDraw $(\log \mathrm{P}=0.76$ and $\log \mathrm{S}=-$ 7.461). Nevertheless, it followed slow kinetics with a Hill slope of $0.9 \pm 0.3$.

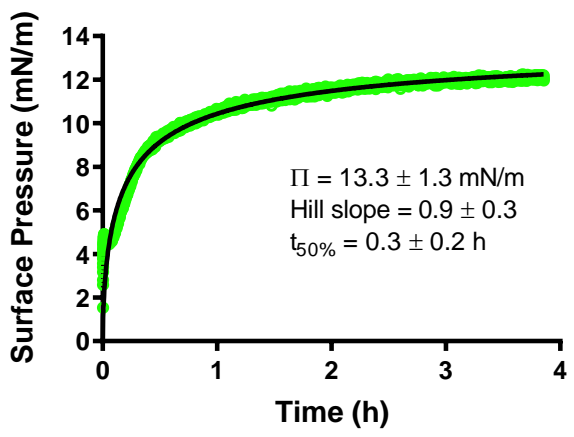

Figure S 7. Representative adsorption isotherms at $23 \pm 1{ }^{\circ} \mathrm{C}$ of PPA148 $(2 \mu \mathrm{g} / \mathrm{mL})$ using $1 \mathrm{mM}$ $\mathrm{MgCl}_{2}$ as a subphase. The black lines are the fitted curves based on the mathematical Hill plot equation. 
Biophysical investigation of the partition of the individual formulation components into the model bacterial IM and OM

Interaction with model OM using Gibbs isotherms

(A)

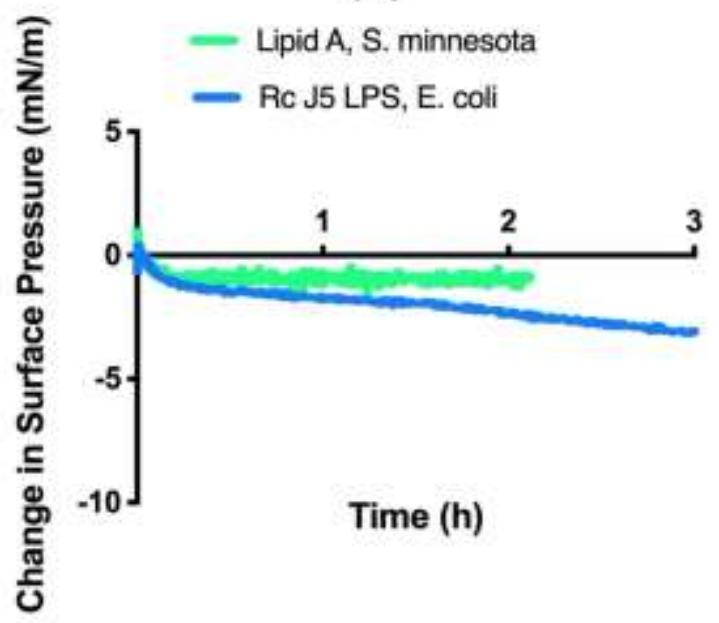

(B)

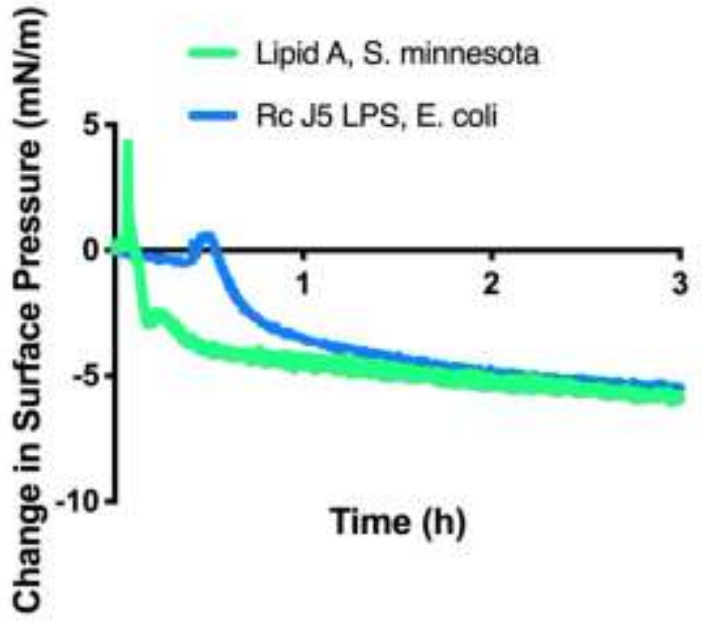

Figure S 8. Representative Gibbs isotherms at $23 \pm 1{ }^{\circ} \mathrm{C}$ of (A) HP $\beta C D$ and (B) RAMEB using R595 Lipid A extracted from Salmonella minnesota and Rc J5 LPS extracted from E. coli monolayers and $1 \mathrm{mM} \mathrm{MgCl} 2$ as a subphase. Both Lipid A and LPS monolayers were compressed at a surface pressure of $30-35 \mathrm{mN} / \mathrm{m}$ and the CDs were used at a concentration of $1 \%$.

\section{Solid-supported asymmetric DPPC and LPS bilayer (model OM) deposition}

Three isotherm cycles were conducted prior to deposition to examine the stability of the monolayers. For the LB deposition, the silicon block was submerged into the ultrapure nonbuffered water subphase, which contained $5 \mathrm{mM} \mathrm{CaCl}_{2}$ and was cooled to $10^{\circ} \mathrm{C}$ to improve interfacial coverage. 
A

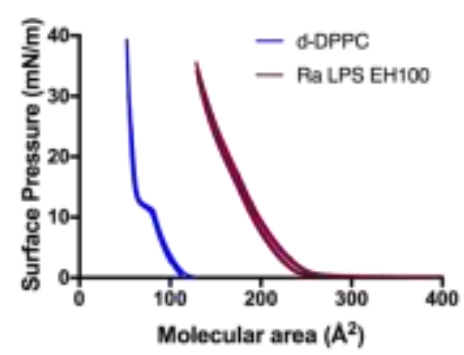

B

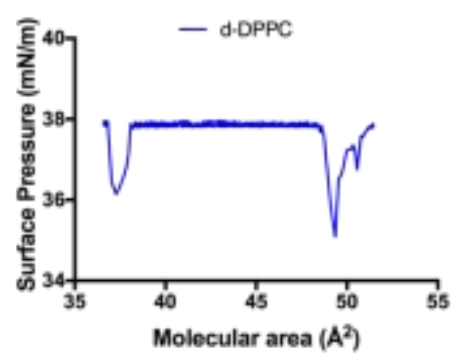

C

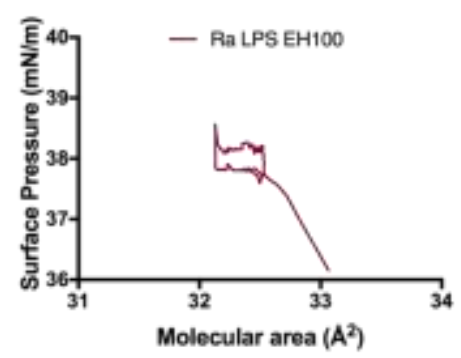

Figure S 9. (A) Langmuir trough isotherm cycles for $\mathrm{d}_{62} \mathrm{DPPC}$ and $\mathrm{h}$ Ra LPS. Change of surface pressure during application of the Langmuir Blodget (B) and Langmuir-Schaefer (C) technique for the deposition of $\mathrm{d}_{62} \mathrm{DPPC}$ and $\mathrm{h}$ Ra LPS respectively. The subphase of the Langmuir trough contained $5 \mathrm{mM} \mathrm{CaCl}_{2}$ and was cooled to $10^{\circ} \mathrm{C}$ during deposition. 


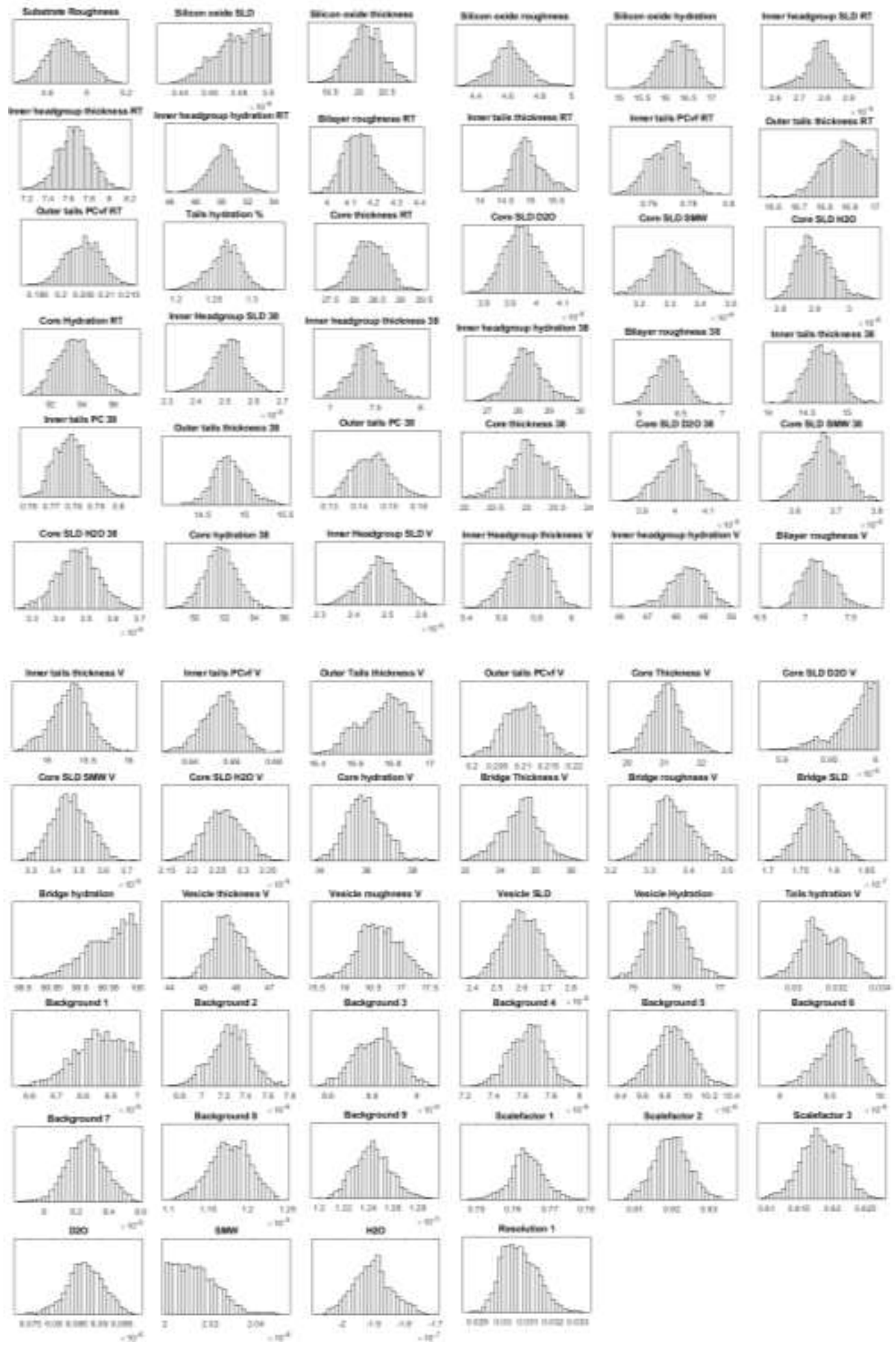

Figure S 10. Posterior distributions for all the layers of the system presented in Figure 9. 
Table S 2. Best fit parameter values with $95 \%$ confidence interval for the fits shown in Figure 9. The minimum and maximum values in parenthesis present the range of the skewed distribution.

\begin{tabular}{|l|l|}
\hline \multicolumn{1}{|c|}{ Parameters } & \multicolumn{1}{|c|}{ Value (min, max) } \\
\hline Substrate Roughness & $4.8991(4.7208,5.0884)$ \\
\hline Silicon_oxide_SLD & $3.4781 \mathrm{e}-06(3.4465 \mathrm{e}-06,3.499 \mathrm{e}-06)$ \\
\hline Silicon_oxide_thickness & $20.13(19.581,20.721)$ \\
\hline Silicon_oxide_roughness & $4.6132(4.3867,4.8586)$ \\
\hline Silicon_oxide_hydration & $16.268(15.565,16.889)$ \\
\hline Inner Headgroup SLD 38 & $2.5114 \mathrm{e}-06(2.3947 \mathrm{e}-06,2.6203 \mathrm{e}-$ \\
\hline Inner headgroup thickness 38 & $06)$ \\
\hline Inner headgroup hydration 38 & $7.4062(7.0304,7.792)$ \\
\hline Bilayer roughness 38 & $28.27(27.132,29.476)$ \\
\hline Inner tails thickness 38 & $6.3523(5.9945,6.6972)$ \\
\hline Inner tails PC 38 & $14.7(14.316,15.103)$ \\
\hline Outer tails thickness 38 & $0.77944(0.76626,0.79521)$ \\
\hline Outer tails PC 38 & $14.835(14.435,15.294)$ \\
\hline Core thickness 38 & $0.14432(0.13359,0.15699)$ \\
\hline Core SLD D2O 38 & $23.063(22.359,23.684)$ \\
\hline Core SLD SMW 38 & $4.01 \mathrm{e}-06(3.8949 \mathrm{e}-06,4.1266 \mathrm{e}-06)$ \\
\hline Core SLD H2O 38 & $3.6736 \mathrm{e}-06(3.584 \mathrm{e}-06,3.7649 \mathrm{e}-06)$ \\
\hline Core hydration 38 & $3.47 \mathrm{e}-06(3.3117 \mathrm{e}-06,3.6208 \mathrm{e}-06)$ \\
\hline & $51.774(49.615,54.063)$ \\
\hline
\end{tabular}




\begin{tabular}{|l|l|}
\hline Inner Headgroup SLD V & $2.48 \mathrm{e}-06(2.3597 \mathrm{e}-06,2.5928 \mathrm{e}-06)$ \\
\hline Inner Headgroup thickness V & $5.7482(5.5157,5.9499)$ \\
\hline Inner headgroup hydration V & $48.437(47.038,49.648)$ \\
\hline Bilayer roughness V & $7.1503(6.8428,7.5363)$ \\
\hline Inner tails thickness V & $15.284(14.811,15.713)$ \\
\hline Inner tails PC V & $0.65456(0.63677,0.67044)$ \\
\hline Outer Tails thickness V & $16.759(16.504,16.97)$ \\
\hline Outer tails PC V & $0.20992(0.20252,0.21703)$ \\
\hline Core Thickness V & $21.052(20.16,22.068)$ \\
\hline Core SLD D2O V & $5.9742 \mathrm{e}-06(5.9184 \mathrm{e}-06,5.9992 \mathrm{e}-$ \\
\hline Core SLD SMW V & $06)$ \\
\hline Core SLD H2O V & $3.4677 \mathrm{e}-06(3.319 \mathrm{e}-06,3.6196 \mathrm{e}-06)$ \\
\hline Core hydration V & $2.263 \mathrm{e}-06(2.1871 \mathrm{e}-06,2.3389 \mathrm{e}-06)$ \\
\hline Bridge Thickness V & $35.969(34.486,37.55)$ \\
\hline Bridge roughness V & $34.594(33.428,35.743)$ \\
\hline Bridge SLD & $3.3608(3.262,3.4725)$ \\
\hline Bridge hydration & $07.7754 \mathrm{e}-07(1.7224 \mathrm{e}-07,1.8266 \mathrm{e}-$ \\
\hline Vesicle thickness V & $99.944(99.851,99.997)$ \\
\hline Vesicle roughness V & $45.821(44.892,46.882)$ \\
\hline Vesicle SLD & $16.624(15.915,17.345)$ \\
\hline
\end{tabular}




\begin{tabular}{|l|l|}
\hline Vesicle Hydration & $75.799(75.023,76.735)$ \\
\hline Tails hydration V & $0.031375(0.029517,0.033136)$ \\
\hline D2O & $\begin{array}{l}6.0869 \mathrm{e}-06(6.0783 \mathrm{e}-06,6.0944 \mathrm{e}- \\
06)\end{array}$ \\
\hline SMW & $2.014 \mathrm{e}-06(2.0006 \mathrm{e}-06,2.0346 \mathrm{e}-06)$ \\
\hline H2O & $-1.9024 \mathrm{e}-07(-2.0229 \mathrm{e}-07,-1.7735 \mathrm{e}-$ \\
& $07)$ \\
\hline Resolution & $0.030681(0.029465,0.032202)$ \\
\hline
\end{tabular}

